\title{
Hypocomplementemic urticarial vasculitis
}

INSERM

\section{Source}

INSERM. (1999). Orphanet: an online rare disease and orphan drug data base.

Hypocomplementemic urticarial vasculitis. ORPHA:36412

Hypocomplementemic urticarial vasculitis (HUV) is an immune complex-mediated small vessel vasculitis characterized by urticaria and hypocomplementemia (low C1q with or without low (3 and (4), and usually associated with circulating anti-C1q autoantibodies. Arthritis, pulmonary disease, ocular inflammation, and glomerulonephritis are common systemic manifestations. 